\title{
PERFIL DO CONSUMIDOR DE FRUTAS EM CIDADES DO INTERIOR DO ESTADO DE SÃO PAULO - SP'
}

\author{
JOSÉ MATHEUS YALENTI PEROSA², MARIA APARECIDA ANSELMO TARSITANO, \\ MARIA INÊZ ESPAGNOLI GERALDO MARTINS ${ }^{4}$, \\ GESSUIR PIGATTO ${ }^{5}$, ALESSANDRO ANTONANGELO 6
}

RESUMO- Estudos relacionados ao perfil do consumidor colocam-se como importante subsídio à participação competitiva dos diferentes segmentos nas cadeias agroalimentares e para a própria organização da mesma. $\mathrm{O}$ objetivo do presente trabalho foi o de identificar o perfil dos consumidores de frutas em cidades do interior do Estado de São Paulo. A amostra foi constituída de 404 consumidores em pontos de venda previamente selecionados nas cidades de Botucatu, Avaré, Jaboticabal, Ilha Solteira e Tupã. Os resultados sobre o perfil do consumidor de frutas nas cidades pesquisadas sinalizam uma preocupação com as características do produto (saudáveis e saborosos), com o preço e com facilidades de acesso na compra. Os resultados apontam também para a necessidade de maiores estudos sobre a importância da informação relacionada aos aspectos mais valorizados (características do produto e preço) na estrutura de consumo.

Termos para indexação: perfil do consumidor; frutas; Estado de São Paulo.

\section{FRUIT CONSUMER PROFILE IN CITIES OF THE INTERIOR OF THE STATE OF SÃO PAULO - SP}

\begin{abstract}
Scientific studies analyzing consumers profile and decision-making processes play an important role on companies' strategies and vertical coordination action to increase efficiency on food chains. This research mapped the profile of food consumers on medium size cities on the state of Sao Paulo, Brazil. The sample considered 404 consumers in sale spots of 5 cities: Botucatu, Avaré, Jaboticabal, Ilha Solteira and Tupã. The results suggested that consumers of the 5 cities are concerned about fruit attributes like healthiness and flavor, but not neglecting price and access to market facilities. These results highlighted the importance of marketing actions to emphasize important characteristics valued by consumers, like price and product related attributes. Index terms: consumer profile, fruits, marketing, state of São Paulo, Brazil.
\end{abstract}

\section{INTRODUÇÃO}

O processo de liberalização comercial e de integração de mercados tem impactado fortemente as economias nacionais de todo o mundo.

A forte concorrência advinda deste processo tem induzido agentes econômicos de cadeias agroalimentares a uma conduta mais eficiente, visando a uma inserção competitiva nas mesmas. Neste ambiente de mercado, questões como diferenciação de produtos e hábitos de consumo têm-se colocado como balizadores importantes de estudos, subsidiando políticas públicas e investimentos privados.

As transformações no consumo de alimentos têm-se acentuado nas últimas décadas. Uma das características mais visíveis dessa transformação é a estreita relação entre consumo de alimentos e a questão da saúde (BARROS et al., 2007).
Nessa tendência, mesmo com preços superiores aos similares tradicionais, produtos light ou diet vêm ganhando espaço nas vendas. Da mesma forma, o consumo de frutas vem ganhando terreno nas preferências do consumidor. Segundo recente estudo do CEPEA/ESALQ, ao contrário do consumo per cápita de hortaliças, que passou de 29 para 27 quilos no período entre 2002 e 2008, o consumo de fruta passou de 24 para 28 quilos (SILVEIRA et al., 2011). No entanto, novos hábitos de consumo colocam- se como obstáculos a essa tendência: estudo mostra que entre os consumidores que se alimentam fora de casa com regularidade, apenas $4 \%$ das pessoas incluem frutas na alimentação, percentual bem inferior ao obtido na alimentação no lar (37\%) (SEBRAE, 2012).

Estudos sobre o comportamento do consumidor são fundamentais para compreender as demandas do consumidor, permitindo que todos os integrantes

\footnotetext{
1(Trabalho 255-11). Recebido em: 07-11-2011. Aceito para publicação em: 05-09-2012.

${ }^{2}$ Prof. da Faculdade de Ciências Agronômicas-Câmpus de Botucatu/UNESP. E-mail: dede@fca.unesp.br

${ }^{3}$ Profa. da Faculdade de Engenharia - Câmpus de Ilha Solteira/UNESP. E-mail: maat@agr.feis.unesp.br

${ }^{4}$ Profa. da Faculdade de Ciências Agrárias e Veterinárias - Câmpus de Jaboticabal/UNESP. E-mail: minezesp@fcav.unesp.br

${ }^{5}$ Prof. do Câmpus Experimental de Tupã/UNESP. E-mail: pigatto@tupa.unesp.br

${ }^{6}$ Prof. da Faculdade do Sudoeste Paulista - Avaré. E-mail alessandro@fspnet.com.br.
} 
dos sistemas agro- alimentares adaptem suas estratégias. Inúmeros estudos (PEROSA et al., 2006; SOUZA et al., 2008; TREVISAN et al., 2010) têm-se dedicado a pesquisar o perfil do consumidor e os critérios para a decisão de compra no setor frutícola.

Nesse mercado, essa questão reveste-se de importância, dadas as características diferenciadas da produção, os inúmeros arranjos organizacionais possíveis para levar o produto até o consumidor, assim como as transformações verificadas na demanda em todo o mundo. Nesse sentido, estudos relacionados ao perfil da demanda de frutas colocam-se como importante subsídio à participação competitiva dos agentes na cadeia e da própria organização da mesma.

\section{Objetivo geral}

Caracterizar o perfil dos consumidores de frutas em cidades do interior do Estado de São Paulo, como subsídio à elaboração de estratégias de participação de diferentes agentes da cadeia no mercado.

Objetivos especificos: identificar as principais frutas consumidas e locais de compra; levantar as principais razões para o consumo de frutas; verificar os principais atributos das frutas priorizados pelos consumidores; delimitar e analisar a relação entre o tipo de fruta consumida, as diferentes faixas etárias, o local de compra e o grau de instrução dos consumidores.

\section{MATERIAL E MÉTODOS}

Estudos sobre o comportamento do consumidor contribuem para a compreensão de como funciona as decisões de compra e o processo de geração de valor para o consumidor.

Perosa et al. (2009) destacam 5 abordagens sobre o comportamento do consumidor: a) Teoria da Racionalidade Econômica; b) Teoria Comportamental; c) Teoria Psicanalítica; d) Teoria Social e Antropológica; e) Teoria Cognitiva.

Dentre os teóricos que estudaram as motivações dos indivíduos, destaca-se Maslow, que apresentou uma escala hierárquica de níveis de necessidade. A Hierarquia de Maslow, citado por Mowen e Minor (2003), classificam-os de Fisiológicas (nível 7) a Autorrealização (nível 1). Os indivíduos buscam satisfazer suas necessidades mais básicas e objetivas, como a alimentação (nível 7 da hierarquia), e as necessidades mais complexas e subjetivas que atendam a desejos pessoais e ideológicos. Essas necessidades seriam satisfeitas de acordo com as possibilidades dos indivíduos.

Suportado pela Teoria Cognitiva e pelo seu desdobramento por Maslow, o 'marketing mix' é uma ferramenta que tem sido usada em pesquisas empíricas e permite a compreensão de desejos e necessidades dos consumidores. Muito utilizado por empresas para elaboração de planejamento estratégico de mercado, a ferramenta é composta por quatro elementos, formando o Composto de Marketing ou Marketing Mix: produto, preço, promoção e praça, também conhecidos como 4Ps. A interação destes elementos é importante para que a organização alcance seus objetivos em consonância com a satisfação de necessidades e desejos dos consumidores. A Teoria Cognitiva e seus desdobramentos permitem uma análise mais detalhada do processo decisório que orienta o consumo de frutas e dá suporte ao presente estudo.

O método de pesquisa foi o exploratório, tendo-se adotado uma amostra não probabilística. Procurou-se, no entanto, delimitar um número mínimo de entrevistados (entre 0,1 a $0,2 \%$ da população de cada cidade pesquisada), delimitação da coleta nos principais locais de venda em bairros de diferentes classes de renda. Realizaram-se também entrevistas em diferentes horários nos locais de venda (pela manhã, à tarde e no período noturno), visando a entrevistar uma gama mais diversificada de consumidores. Para a seleção dos pontos de venda a serem pesquisados, foi realizada uma avaliação prévia daqueles mais importantes na comercialização de frutas nas cidades participantes do estudo.

A amostra foi composta por 404 consumidores em pontos de venda, nas cidades de Botucatu, Avaré, Jaboticabal, Ilha Solteira e Tupã, e a coleta dos dados foi realizada no primeiro semestre de 2008 .

O instrumento utilizado para a coleta dos dados foi um questionário composto por questões fechadas. A identificação das razões de consumo foi elaborada com base nos conceitos de marketing estratégico. Foram formuladas 20 assertivas, ordenadas de acordo com o composto de marketing: preço, produto, promoção e pontos de venda, e colocadas em uma escala ordinal, tipo Likert, composta por cinco categorias de respostas. A escala Likert é uma escala de resposta usada habitualmente em pesquisas de opinião. Os consumidores entrevistados devem especificar seu nível de concordância com uma afirmação. Normalmente, o que se deseja medir é o nível de concordância ou não concordância à afirmação. As respostas apontam a intensidade crescente com que a assertiva apresentada influencia a decisão de consumo pelo respondente (LIKERT, 1971).

Após as entrevistas, os dados recolhidos foram tabulados no software Microsoft Excel for Windows, sendo submetidos à estatística descritiva e sistematizados em gráficos e tabelas para análises. 


\section{RESULTADOS}

Em relação ao gênero, os consumidores entrevistados estão representados com aproximadamente metade do sexo masculino e metade do sexo feminino. Entre as cidades, essa proporção é relativamente próxima da média geral. Os consumidores entrevistados, na sua grande maioria, estão entre $21 \mathrm{e}$ 50 anos de idade, com pequena participação dos mais jovens (4,5\%). Essa constatação reflete o fato de que quem realiza as compras de frutas são pessoas mais velhas. As maiorias dos consumidores entrevistados têm curso secundário e superior (78\%). É importante destacar essa constatação, uma vez que o hábito de consumir frutas pode ser formado espontaneamente, ou pelo acesso a informações sobre as quais instrução pode ter influência.

Quando perguntados sobre os meios de comunicação mais usados para ter informações sobre os produtos consumidos, a grande maioria dos entrevistados $(65 \%)$ tem acesso a informações através da mídia Televisão/Internet. Se no caso da televisão o grau de instrução pouca influência tem, o uso da Internet pode pressupor uma instrução maior, dado o conhecimento necessário para seu uso. Do total de consumidores de frutas que se utilizam dessa mídia, $31 \%$ declararam que o acesso a informações sobre os produtos consumidos é realizado via Internet.

A Tabela 1 mostra diferentes influências na aquisição do hábito de consumo. O somatório da pontuação e a participação relativa das principais influências na aquisição do hábito de consumo de frutas são apresentados nesta tabela.

Cerca de $37 \%$ dos entrevistados declararam ter adquirido o hábito espontaneamente, seguido pela influência dos pais (35\%). Na amostra entrevistada, a influência da escola e da mídia tem menor peso. Infere-se pelas informações que os consumidores são mais sensíveis à influência dos pais e, mesmo buscando informações na mídia, acreditam que a aquisição do hábito se verifica de forma espontânea, sem uma influência maior da mesma.

A periodicidade do consumo de frutas é diária para a maioria dos entrevistados $(56 \%)$ e semanal (30\%). Ressalte-se uma diferença significativa entre as cidades: enquanto $66 \%$ dos consumidores de Jaboticabal consomem frutas diariamente, apenas $44 \%$ dos consumidores de Tupã o fazem. Nesse aspecto, destaque-se Ilha Solteira em relação às demais cidades, onde um percentual elevado de consumidores $(16 \%)$ declararam consumir frutas raramente.

Esse consumo se verifica na forma natural (69\%), na forma de sucos $(25 \%)$, em saladas $(2 \%)$ e doces (4\%). Embora a logística seja importante para disponibilizar ao consumidor final um produto com qualidade sob qualquer forma, essa questão se coloca como muito importante para o consumo in natura, dada a perecibilidade da maioria das frutas.

As frutas mais consumidas são mostradas na Figura I: outras frutas não quantificadas, como manga e pera, foram mencionadas como de consumo relevante nas entrevistas.

As principais frutas consumidas foram banana, laranja e maçã, seguida pelo mamão. Esse consumo segue o padrão da região Sudeste: de acordo com a Pesquisa de Orçamentos Familiares - POF/ IBGE, e na região Sudeste as frutas mais consumidas foram banana, laranja, maçã e mamão (IBGE, 2010).

Destaca-se o consumo de maçã, superior ao de outras frutas mais tradicionais, como abacaxi e mamão. Questões relativas à oferta desta fruta durante todo o ano, preços acessíveis, praticidade para o consumo individual e facilidade de acesso, são razões que podem ser apontadas para esse consumo.

Em relação aos locais de compra, destaca-se o supermercado como principal local de compras em quase todas as cidades, seguido por quitandas e feiras. No caso dos equipamentos feiras e quitandas, observa-se uma diferença de sua importância entre as cidades: enquanto as quitandas não têm importância na cidade de Ilha Solteira (não havia esse equipamento voltado ao comércio de frutas), as feiras constituem o principal local de compra. Já em Jaboticabal, esse equipamento é pouco significativo. Esses dados mostram a necessidade de se pensar localmente as estratégias de distribuição de frutas nas diferentes cidades.

Na Tabela 2, são apresentadas as razões que motivam o consumo de frutas pelos entrevistados.

Destacam-se como motivos relevantes de consumo os itens 1, 4,11, 13 e 16, com média entre 4 e 5. Essas questões estão relacionadas com o produto em si (saborosos, fazem bem à saúde, alimentos funcionais, etc.). Foi destacada, também, uma questão relacionada ao ponto de venda (item 4).

O segundo bloco de razões valorizadas pelo consumidor (média entre 3 e 4) está relacionado à dimensão preço (item 9 e 12), à qualidade do produto consumido (itens 2,20 ) e, novamente, à dimensão pontos de venda (itens 7, 15 e 19).

Como razões de menor influência no consumo (média abaixo de 3 ), aparecem os de itens $2,3,5,6,8,10,14,17,18$ e 19 .

Embora o preço seja lembrado, tanto no grupo de razões mais valorizadas, como no intermediário, destaca-se no perfil do consumidor de frutas, em todas as cidades, preocupação relacionada ao produto e, em menor escala, aos pontos de venda. 
Embora haja uma valorização média padrão, verificam-se algumas diferenças nas cidades pesquisadas: em Jaboticabal e Tupã, foi bem valorizada a questão da existência de pontos de venda de fácil acesso (item 7), bem como a existência de grande variedade de frutas e alimentos no local de venda (item 15). Essa preocupação relacionada aos pontos de venda também aparece no item 19 (Comodidade de se consumir) em Tupã e, também, em Jaboticabal.

Essas informações apontam que o acesso ao consumo de frutas nessas praças não constitui uma preocupação do consumidor, questão essa merecedora de mais atenção em outras cidades.

Por último, a Tabela 3 mostra a visão do consumidor de cada cidade pesquisada sobre a melhor combinação de atributos relacionados ao preço, praça, produto e promoção. Foi solicitado ao entrevistado valorizar numa escala de 1 a 5 , a combinação de fatores que melhor definiram seu comportamento de compra. Os fatores apresentados foram relativos às características do produto consumido, ao preço, ao acesso aos locais de compra, e às informações promocionais (como marca e certificação).
Observa-se, na tabela, uma relativa uniformidade na combinação dos atributos valorizados nas diferentes cidades.

O principal atributo valorizado para o consumo foi o produto, suas características intrínsecas . O segundo atributo valorizado foi relativo ao preço, seguido pela facilidade de acesso na compra do produto.

$\mathrm{O}$ atributo relacionado à promoção/informação do produto (marca e certificação) aparece com $27 \%$ das respostas. Essa valorização pelo consumidor requer uma consideração: a compreensão do significado de marca/certificação para o entrevistado pode ter sido distinta daquela pretendida pelo entrevistador. É possível que o entrevistado, no ato de valorizar a melhor combinação, tenha considerado "marca/ certificação" em grande parte como um atributo da qualidade do produto, não relacionado diretamente com a promoção do mesmo. Nesse sentido, a combinação de atributos teria um peso maior para o produto e menor para "informação/promoção".

A tabela aponta para uma característica do perfil do consumidor de frutas, valorizando as características do produto e, em menor escala, as questões relacionadas ao custo.

TABELA 1 - Influência na aquisição do hábito de consumo dos entrevistados em diferentes cidades do Estado de São Paulo em 2008.

\begin{tabular}{lcccccccc}
\hline & Pais & \multicolumn{3}{c}{ Escola } & \multicolumn{2}{c}{ Mídia } & \multicolumn{3}{c}{ Espontâneo } \\
\hline & Pontuação & $\mathbf{\%}$ & Pontuação & $\mathbf{\%}$ & Pontuação & $\mathbf{\%}$ & Pontuação & $\mathbf{\%}$ \\
\hline Botucatu & 477 & 36,4 & 90 & 18,0 & 112 & 20,1 & 421 & 29,9 \\
\hline Ilha Solteira & 126 & 9,6 & 62 & 12,4 & 66 & 11,8 & 169 & 12,0 \\
\hline Jaboticabal & 265 & 20,2 & 169 & 33,9 & 190 & 34,1 & 289 & 20,5 \\
\hline Tupã & 221 & 16,9 & 137 & 27,5 & 134 & 24,1 & 289 & 20,5 \\
\hline Avaré & 221 & 16,9 & 41 & 8,2 & 55 & 9,9 & 241 & 17,1 \\
\hline Total & $\mathbf{1 3 1 0}$ & $\mathbf{1 0 0 , 0}$ & $\mathbf{4 9 9}$ & $\mathbf{1 0 0 , 0}$ & $\mathbf{5 5 7}$ & $\mathbf{1 0 0 , 0}$ & $\mathbf{1 4 0 9}$ & $\mathbf{1 0 0 , 0}$ \\
\hline Média & $\mathbf{3 4 , 7}$ & & $\mathbf{1 3 , 2}$ & & $\mathbf{1 4 , 8}$ & & $\mathbf{3 7 , 3}$ & \\
\hline Fonte: pesquisa de campo & & & & & & \\
\hline
\end{tabular}




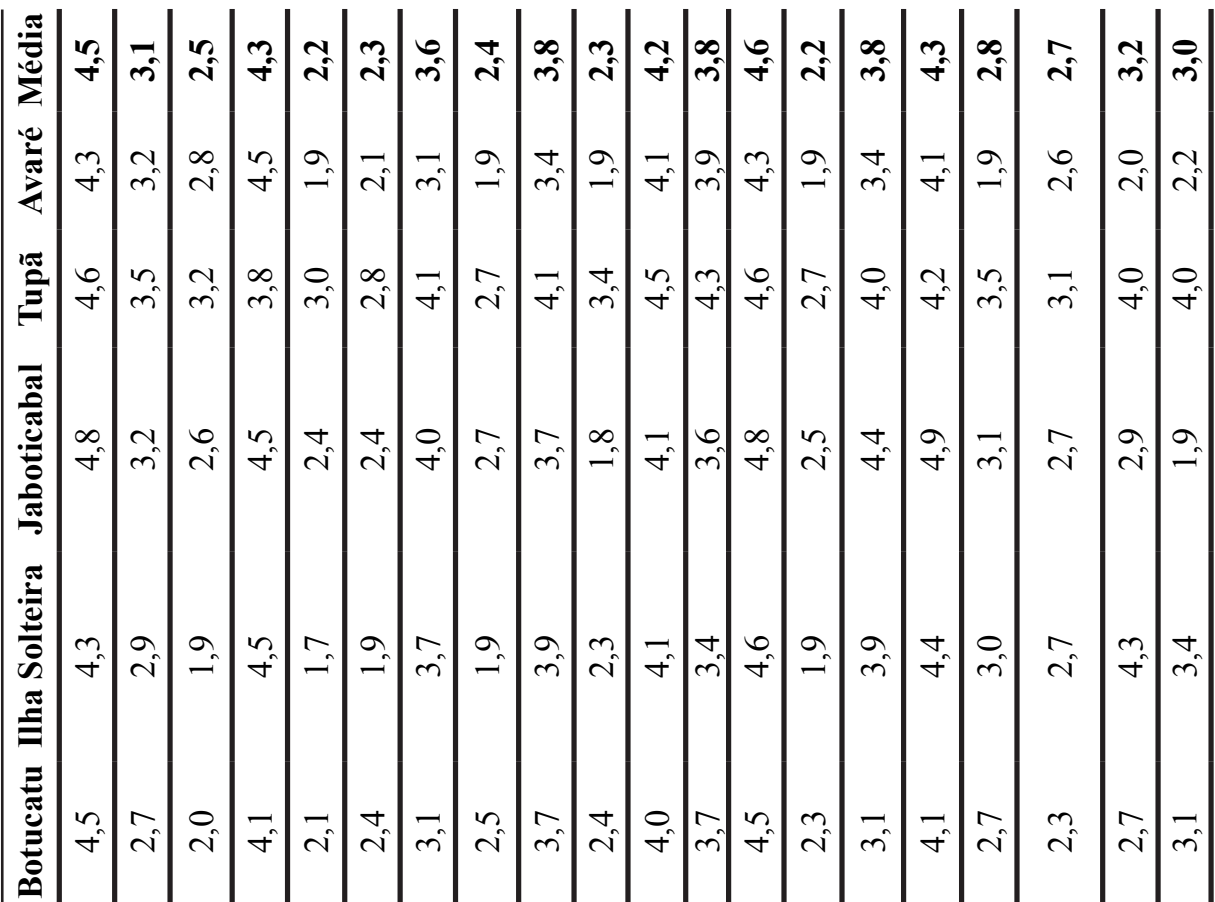


TABELA 3 - Melhor combinação de atributos para o consumo de fruta dos entrevistados em diferentes cidades do Estado de São Paulo em 2008.

\begin{tabular}{|c|c|c|c|c|c|c|c|c|c|c|}
\hline Cidades & Produto & & Marca & & Certificação & & Acesso & & Preço & \\
\hline & $\%$ & $\mathrm{n}^{\mathrm{o}}$ & $\%$ & $n^{\circ}$ & $\%$ & $\mathrm{n}^{\mathrm{o}}$ & $\%$ & $n^{\circ}$ & $\%$ & $\mathrm{n}^{\mathrm{o}}$ \\
\hline Botucatu & 30,3 & 653 & 14,0 & 302 & 14,6 & 314 & 18,2 & 392 & 22,9 & 493 \\
\hline Ilha Solteira & 27,4 & 178 & 12,8 & 83 & 12,3 & 80 & 20,5 & 133 & 27,0 & 175 \\
\hline Jaboticabal & 26,0 & 281 & 18,1 & 196 & 15,1 & 163 & 18,7 & 202 & 22,0 & 238 \\
\hline Tupã & 27,9 & 264 & 13,8 & 130 & 12,6 & 119 & 23,8 & 225 & 21,9 & 207 \\
\hline Avaré & 30,5 & 362 & 15,1 & 179 & 11,8 & 140 & 19,1 & 226 & 23,5 & 278 \\
\hline Média & 28,4 & 1738 & 14,8 & 890 & 13,3 & 816 & 20,1 & 1178 & 23,5 & 1391 \\
\hline
\end{tabular}

Fonte: Pesquisa de campo.

\section{CONCLUSÕES}

Três aspectos se destacam no estudo:

- Os consumidores entrevistados estão concentrados na faixa etária acima de 21 anos de idade. Essa faixa etária tem um consumo proporcionalmente maior de laranja, banana, maçã e mamão, e preferem supermercados e feiras como locais de compra.

- A grande maioria consome frutas em seu estado natural e apenas $25 \%$ na forma de sucos, e valoriza aspectos relativos ao produto em si.

- As razões para o consumo de frutas estão ligadas ao sabor, à saúde e à qualidade da fruta $\mathrm{e}$ do local onde são adquiridas; os preços também influenciam muito na hora da compra.

Em relação à primeira consideração, os consumidores com mais de 50 anos têm a mesma preferência que aqueles da faixa etária entre $21 \mathrm{e}$ 50 anos. Considerando o envelhecimento natural da população, essa constatação aponta, no futuro, para uma escolha de frutas semelhante à atual.

Em relação à forma de consumo de frutas, a alteração na distribuição de renda no País tem propiciado maior participação das classes $\mathrm{C}$ e $\mathrm{D}$ entre os consumidores. Em recente pesquisa sobre o perfil dos consumidores (SEBRAE, 2012), verificou-se que os gastos com alimentação fora de casa têm-se direcionado a produtos processados/industrializados. Nesse sentido, é de se esperar um crescimento de frutas processadas, como sucos, doces e outros.

As diferentes informações sobre o perfil do consumidor de frutas nas cidades pesquisadas sinalizam uma preocupação com as características do produto (saudáveis e saborosos), com o preço e com as facilidades de acesso na compra. Por outro lado, questões relativas a informações sobre o produto não foram tão valorizadas (marca e certificação como motivos de compra).
Tal perfil constitui importante indicativo para o planejamento de vendas no varejo da cidade, bem como para os demais elos da cadeia, produção e intermediação.

Tais resultados apontam para a necessidade de maiores estudos sobre a importância da informação relacionada aos aspectos mais valorizados (características do produto e preço) na estrutura de consumo.

\section{REFERÊNCIAS}

BARRoS, M. A. B.; LOPES, G. M .B.; WANDERLEY, M. B. Tipologia do consumo de frutas: um estudo sobre o comportamento do consumidor de banana. Revista Produção On Line, Florianópolis, 2007. Disponível em: $<\underline{w w w}$. producaoonline.ufsc.br>. Acesso em: 12 jan. 2011.

IBGE - Instituto Brasileiro de Geografia e Estatística. Pesquisa de orçamentos familiares 2008/2009. Rio de Janeiro, 2010.

LIKERT, R. Novos padrões de administração. São Paulo: Atlas, 1971.

MOWEN, I. C.; MINOR, M. S. Comportamento do consumidor. São Paulo: Prentice Hall, 2003. 403 p.

PEROSA, J. M. Y.; VIEIRA, E. M.; NITZSCHE, T. Cadeia produtiva da nêspera na região do Alto Tietê: indicadores econômicos da produção e mercado atacadista. Revista Brasileira de Fruticultura, Jaboticabal, v. 28, p. 214-217, 2006. 
PEROSA, J.M.Y.; MOORI, R.G.; LOMBARDI, M.F.S.; PEROSA, B.B. O Estímulo Local e o Consumo de Produtos Orgânicos em Botucatu-SP. Revista de Estudos Sociais, Cuiabá, v.11, n. 22, 2009.

SEBRAE. Boletim de tendências. Disponível em: $<$ www.sebrae.com.br/setor/fruticultura $>$. acesso em: 15 ago 2012.

SILVEIRA, J.; GALESKAS, H.; TAPETTI, R; LOURENCINI, I. Quem é o consumidor brasileiro de frutas e hortaliças? Revista Hortifruti Brasil, São Paulo, p. 8-23, 2011. SOUZA, R. S.; ARBAGE, A. P.; NEUMANN, P. S.; FROEHLICH J. M.; DIESEL, V.; SILVEIRA, P. R.; SILVA, A.; CORAZZA, C.; BAUMHARDT, E.; LISBOA, R. S. Comportamento de compra dos consumidores de frutas, legumes e verduras na região central do Rio Grande do Sul. Ciência Rural, Santa Maria, v. 38, n.2, p. 11-517, 2008.

TREVISAN, R.; PIANA, C.F.B.; TREPTOW, R.O.; GONÇALVES, E.D.; ANTUNES, L.E.C.. Perfil e preferências do consumidor de pêssego (Prunus persica) em diferentes regiões produtoras no Rio Grande do Sul. Revista Brasileira de Fruticultura, Jaboticabal, v.32, n.1, p. 8-23, 2010. 